\title{
EVALUATION OF VACCINE-INDUCED MATERNAL IMMUNITY AGAINST CLASSICAL SWINE FEVER
}

\author{
P. Soós ${ }^{1 *}$, M. MOJZIS ${ }^{2}$, A. POLLNER ${ }^{3}$ and L. SÜMEGHY ${ }^{3}$ \\ ${ }^{1}$ Department of Microbiology and Infectious Diseases, Faculty of Veterinary Science, \\ Szent István University, H-1581 Budapest, P.O. Box 22, Hungary; ${ }^{2}$ State Veterinary \\ Institute, Zvolen, Slovakia; ${ }^{3}$ Agricultural State Farm, Mezőhegyes, Hungary
}

(Received April 10, 2000; accepted May 3, 2000)

The vaccine-induced maternal immunity against classical swine fever (CSF) was investigated in this study. Eight sows were vaccinated with the Chinese strain of classical swine fever virus (CSFV). The length of time between vaccination and farrowing was 167-217 days. Milk samples from the front, middle and back udder sections and blood samples were taken from the sows on days 3 and 14 after farrowing. Blood samples were obtained from the piglets at the age of 3, 6 and 10 weeks. The antibody level of the milk was examined by ELISA, and that of blood samples by the virus neutralization (VN) test as well. In all 3week-old piglets and in $80 \%$ of the 6 -week-old animals the neutralizing antibody level reached the titre of 1:40. In none of the 10-week-old piglets did the titre exceed the value of 1:20, but in about $25 \%$ of the piglets it reached 1:20; the half of these piglets came from two litters. In none of the piglets did the antibody level reach the negative threshold in the ELISA test during the study. No significant differences were found between the udder sections in milk antibody level by ELISA.

Key words: Classical swine fever, maternal immunity, colostrum, vaccination

The humoral immune response has a dominant role in the antiviral activity of the immune system against classical swine fever (CSF) infection. Although several vaccines were developed because of the ecological significance of the disease, in 1980 the authorities of the European Community decided to eradicate CSF and imposed a ban on vaccination. Eradication is attempted first of all by stamping out and veterinary administrative measures. Vaccination is allowed in situations where initial control measures failed to stop the spread of the infection. Apart from this, in several countries all over the world protection against the disease is based on vaccination with modified live virus even today.

Although several data were presented about the kinetics and duration of maternal immunity in the past years, certain details of vaccine-induced maternal immunity have remained unclear.

\footnotetext{
"E-mail: soos@novell.vmri.hu; Fax: +36 (1) 251-9260
} 
Precaustra et al. (1978) described that the efficacy of vaccination is inversely proportional to the level of maternal antibodies present at the time of the vaccination. In other studies about $95 \%$ of pigs could be immunized very well at neutralization titres of $<1: 32$, while maternal antibodies present in titres higher than 1:64 inhibited the effect of the vaccination. The half-life of maternal antibodies was found to be 12 days (Kim et al., 1984; Suvintarakorn et al., 1993).

Piglets from non-immune sows could be successfully vaccinated from the age of 7 days, while piglets from sows vaccinated 10 months before farrowing could be immunized as early as 5 weeks (Precaustra et al., 1983). Other authors suggest that, in order to avoid the inhibitory effect of maternal antibodies and reach a good immune response, piglets from immune sows should be vaccinated at the age of 7-9 weeks (Terpstra and Wensvoort, 1987).

The antibody level against CSF virus in the colostrum was found to be 24 times higher than that in the serum of the sow and it was even exceeded by the antibody level in the serum of the majority of the piglets. The antibody titres reached the peaks in the piglets between the 9th and 24th hour of life (Lai et al., 1986; Lai et al., 1989).

In challenge studies a neutralization titre higher than 12.5 was sufficient to protect, but virus excretion was abolished only if the titre was $\geq 32$. The threshold for a positive neutralization test in the serological screening of herds is a titre $\geq 1: 25$ (Terpstra and Wensvoort, 1988).

The aim of the present work was to examine the length of protection provided by maternal antibodies in a model experiment. The results are expected to be of use in case of an emergency vaccination, and it is necessary to know how long the maternal immunity can affect the results of methods based on antibody detection.

The following questions were to be answered: (i) how long can a maternal immunity persist which can give sufficient protection against clinical symptoms and death, and inhibit virus excretion; (ii) how long can the maternal antibodies cause false reactions in serological tracing; (iii) are there any differences between different udder sections in the antibody level of the colostrum?

\section{Materials and methods}

Animals

Eight Hungarian Large White sows were vaccinated with the Chinese C strain of CSF virus (Suvac ${ }^{\mathbb{R}}, 2 \mathrm{ml}$ i.m., Bognár and Mészáros, 1963) virus before service. The animals were obtained from a closed herd and housed in isolation units. The length of time between vaccination and farrowing ranged between 167 and 217 days. 
Blood samples were collected from the sows 3 and 14 days after farrowing and from the 66 piglets at the age of 3, 6 and 10 weeks. Milk samples were taken from the front, middle and back sections of the udder on day 3 and 14 after farrowing. CSF antibody levels in the milk were measured by ELISA and those in the serum samples by ELISA as well as by the virus neutralization (VN) test.

\section{Serology}

ELISA. The ELISA test was performed as described by Have (1984), with the following modifications.

Biotinylated anti-CSF swine IgG was applied to compete with the test sera for CSF virus antigen binding. The amount of the attached antibody was detected with peroxidase-labelled streptavidine and TMB substrate (SIGMA). The antibody activity of test sera was calculated as percentage inhibition:

$$
\frac{\text { (OD neg. ref. }- \text { OD test serum }) \times 100}{\text { OD neg. ref. }- \text { OD background }}
$$

The optical density (OD) of the background was the value obtained with a strong positive serum.

The cut-off level of inhibition, above which the serum was considered to be positive, was determined at $50 \%$, and below $30 \%$ it was regarded as negative.

Neutralizing peroxidase-linked antibody (NPLA) assay. The NPLA test was based on the technique described by Holm Jensen (1981) and modified by Terpstra et al. (1984), with the following modifications.

After incubation the monolayers were rinsed briefly with PBS, dried with bench ventilator and fixed in an oven at $80^{\circ} \mathrm{C}$ for $1 \mathrm{~h}$. The monolayers were then overlaid with $50 \mu \mathrm{l}$ of goat-anti CSF serum diluted 1:400 and incubated for $1 \mathrm{~h}$ at $37^{\circ} \mathrm{C}$. After subsequent washing, staining was carried out with peroxidase-labelled anti-sheep IgG conjugate (SIGMA) diluted 1:1000.

\section{Results}

The neutralizing antibody titres of the vaccinated sows ranged between 80 and $>640$; with a mean titre 340 on day 3 , and 480 on day 14 after farrowing. The 3-week-old piglets from these dams had an arithmetical mean (henceforth: mean) titre of 183.38 (range 40-640). The mean titre for the 6-week-old piglets was 57.86 (range 10-320); only one of these piglets had a titre of 10 and one had a titre of 320. The 10-week-old piglets had a mean titre of 10.54 (range 5-20). The mean values of the NPLA titres for the litters are shown in Fig. 1. 


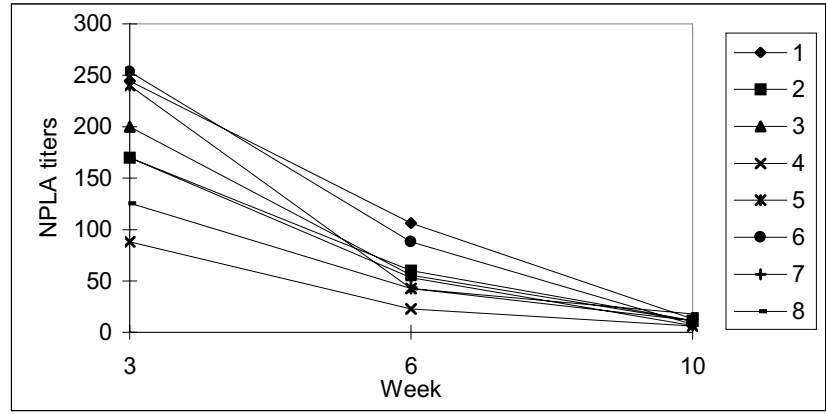

Fig. 1. Mean NPLA titres of the litters of sows 1-8

Table 1

Mean values of the ELISA and NPLA tests

\begin{tabular}{lccc}
\hline \multicolumn{1}{c}{ Animals } & Time & $\begin{array}{c}\text { NPLA mean titres } \\
\text { (range) }\end{array}$ & $\begin{array}{c}\text { ELISA mean values (\%) } \\
\text { (standard deviation) }\end{array}$ \\
\hline \multirow{2}{*}{ Sows } & 3 days & $340(80->640)$ & $\begin{array}{c}101.65(3.67) \\
\end{array}$ \\
& $\begin{array}{c}\text { 14 days } \\
\text { 3 weeks }\end{array}$ & $\begin{array}{c}480.9(8.24) \\
183.38(40-640)\end{array}$ & $101.41(4.00)$ \\
\hline \multirow{2}{*}{ Piglets } & 6 weeks & $57.86(10-320)$ & $92.69(8.65)$ \\
& 10 weeks & $10.54(5-20)$ & $81.45(11.39)$ \\
\hline
\end{tabular}

The mean percentage values obtained by the ELISA test in the serum samples of the sows are summarized in Table 1. The mean ELISA percentage value was 101.41, 92.69 and 81.45 in the 3-week-old, 6-week-old and 10-week-old piglets, respectively. The percentage values obtained by ELISA in the sera of piglets are shown in Fig. 2.

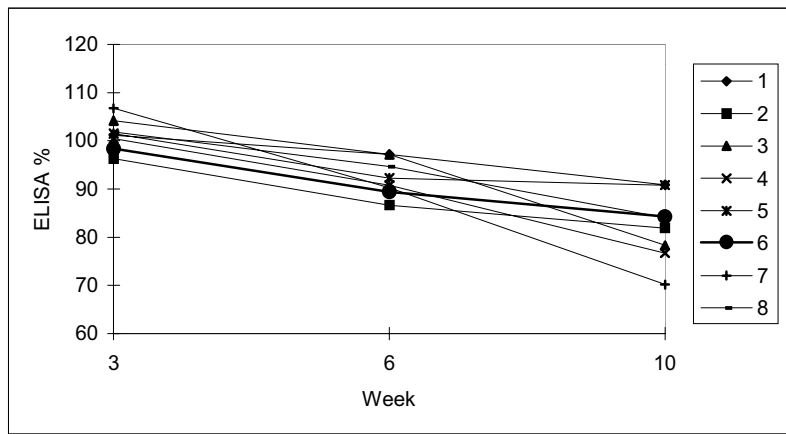

Fig. 2. Mean percentage values obtained in ELISA tests of the sera of piglets in litters 1-8 
The mean ELISA values measured in milk from different udder sections are shown in Fig. 3.

At the age of 3 weeks none of the piglets had an NPLA titre lower than $1: 40$. In $80 \%$ of the piglets the NPLA titre reached or exceeded 1:40 at the age of 6 weeks; only one had a value of 1:10. In 10-week-old piglets the NPLA titre did not exceed 1:20, but in about $25 \%$ of the piglets it reached 1:20; the half of these piglets came from two litters.

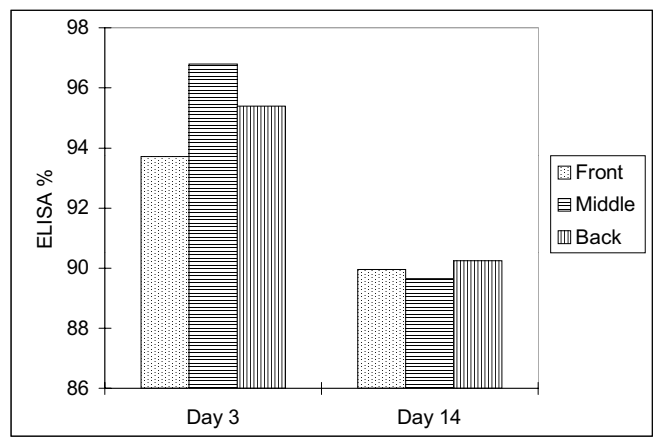

Fig. 3. Mean ELISA values measured in milk from the different udder sections

\section{Discussion}

The vaccination strategy in CSF-endangered areas should not only prevent the clinical cases but also inhibit the shedding of virus. The studies of Terpstra and Wensvoort (1988) showed that NPLA titres $\geq 32$ provide adequate protection to both the individual animal and the herd. They established that NPLA titres $<12.5$ are insufficient to prevent disease and death, however the threshold dilution for a positive NPLA test in serological tracing of infected herds remained 25 , justified by the absence of cross-reactive antibodies against bovine viral diarrhoea virus in pig sera and the initial steep increase of antibody titres after infection with CSF virus strains of low virulence.

This study showed great individual differences in NPLA titres in the litters and among the sows. In all 3-week-old piglets and in $80 \%$ of the 6-week-old animals the neutralizing antibody level reached the titre of 1:40. These animals can be considered as being protected against the infection and, based upon the results of earlier studies, they do not shed the virus. Only one of the 6-week-old pigs failed to reach the titre 1:20, thus the majority of these animals are protected against CSF. This can be stated for only about $25 \%$ of the 10 -week-old piglets; however, a large proportion of them came from two litters.

With regard to the threshold of the positive NPLA test (1:25), the maternal antibodies can be expected not to cause a false positive reaction only if the 
NPLA test was performed from the samples of 10-week-old pigs, because in the majority of the 6-week-old piglets the antibody level reached the titre of 1:40, but in none of the 10-week-old animals did it exceed the titre of 1:20.

The ELISA values reached the threshold of positivity in all piglets during the study period. Thus, in serological screening with this ELISA test a period of 10 weeks is too short for the maternal immunity to reach a level that does not cause false positive reaction.

We could not find any explanation for the observation that the titre of neutralizing antibodies in the serum of some sows increased by day 14. It can be supposed that - because colostrum production has already ceased by that time there is no further excretion of large amounts of antibodies from the blood. It could also be observed that the ELISA value of the milk increased by day 14 in some sows (antibody level increases in the milk and in the serum did not occur in the same sows). Although the level of antibodies did not decrease steeply in the milk (and in some cases, as mentioned above, an increase was measured), the serum antibody level of the piglets decreased during this period, which supports the fact that maternal antibodies can get into the serum only at the beginning of life.

It was described in earlier studies (Kim et al., 1984; Suvintarakorn et al., 1993) that the majority of piglets with NPLA titres lower than 32 could be successfully vaccinated with the $\mathrm{C}$ strain, while titres $>32$ inhibited the effect of the vaccine. In another study (Terpstra and Wensvoort, 1987) the serological response of piglets from immunized sows increased with the age of vaccination from $11 \%$ at $5-6$ weeks to $42 \%$ at $7-8$ weeks and $77 \%$ at $9-10$ weeks. Similarly, we found that $80 \%$ of the piglets had an NPLA titre $\geq 40$ at the age of 6 weeks, while none of the piglets had a titre exceeding 20 at the age of 10 weeks. This suggests that the vaccination of the piglets would be successful at about weeks 7-9 of their life as recommended in the study of Terpstra and Wensvoort (1987).

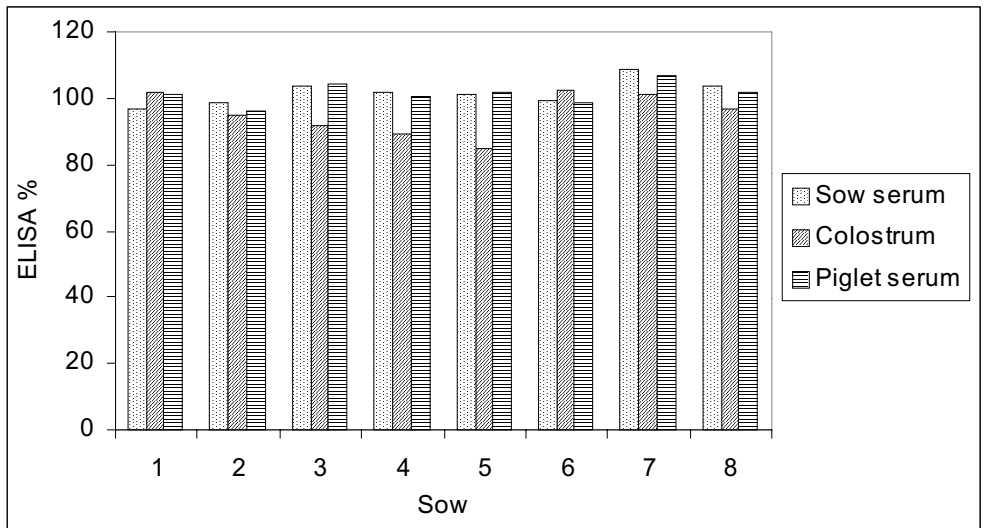

Fig. 4. Comparison of the ELISA values of the sera of piglets (on day 21) with those obtained in the sera and colostrum of the sows (on day 3 ) 
No significant differences were found in the antibody titre of milk from the different udder sections, which is in agreement with the conclusion of Inoue et al. (1980) and Inoue (1981 $a$ and $b$ ), who stated that udder sections had negligible influence on the total IgM, IgG and IgA concentration of swine colostrum.

Lai et al. (1989) described that in sows the neutralizing antibody titre of the colostrum was 2-3 times higher than that of the serum, and most pigs had lower antibody level in the serum than in the colostrum. With the exception of two sows, on day 3 the ELISA values of the colostrum were lower than those obtained in the serum of sows on day 3 and in that of piglets on day 21 (Fig. 4). This may be attributed to the fact that the antibody level of the colostrum was measured only on day 3 after farrowing, by which time it could have decreased.

No correlation was found between the length of time between vaccination and farrowing (167-217 days) and the ELISA value of the milk or the NPLA titre of the sows.

\section{Acknowledgement}

The assistance of the State Veterinary Institute (Zvolen, Slovakia) in the serological examinations is greatly appreciated.

\section{References}

Bognár, K. and Mészáros, J. (1963): Experiences with a 'Chinese' strain of lapinized swine fever virus (in Hungarian, with English abstract). Magyar Állatorvosok Lapja 18, 69-74.

Have, P. (1984): Detection of antibodies against swine fever virus by enzyme-linked immunosorbent assay (ELISA). Acta Vet. Scand. 25, 462-465.

Holm Jensen, M. (1981): Detection of antibodies against hog cholera virus and bovine viral diarrhea virus in porcine serum. A comparative examination using CF, PLA and NPLA assays. Acta Vet. Sand. 22, 85-98.

Inoue, T. (1981a): Possible factors influencing immunoglobulin A concentration in swine colostrum. Am. J. Vet. Res. 42, 533-536.

Inoue, T. (1981b): Possible factors influencing the immunoglobulin M concentration in swine colostrum. Am. J. Vet. Res. 42, 1429-1432.

Inoue, T., Kitano, K. and Inoue, K. (1980): Possible factors influencing the immunoglobulin G concentration in swine colostrum. Am. J. Vet. Res. 41, 1134-1136.

Kim, Y. H., Choi, C. U., Lee, O. S. and Kim, D. W. (1984): Evaluation of a vaccination programme against swine fever in piglets. Research Reports of the Office of Rural Development 26, 53-55.

Lai, S. S., Ho, W. C. and Tsao, S. H. (1986): Absorption of colostral antibodies and neonatal vaccination against hog cholera. J. Chinese Soc. Vet. Sci. 12, 117-123.

Lai, S. S., Lai, L. Y. and Ho, W. C. (1989): Changes of antibody levels in sows influenced by pregnancy. J. Chinese Soc. Vet. Sci. 15, 327-333.

Precaustra, P., Kato, F. and Brun, A. (1978): Peste porcine classique: immunisation active conferee par la souche chinoise CL aux porcelets issus de truies immunes. Dev. Biol. Stand. 41, $367-379$.

Precaustra, P., Kato, F. and Brun, A. (1983): Swine fever; immunisation of piglets. Comp. Immunol. Microbiol. Infect. Dis. 6, 281-289. 
Suvintarakorn, K., Hanveeraphon, A. and Pariyakanok, V. (1993): Efficacy of lapinized swine fever vaccine in piglets born from immune sows. Thai J. Vet. Med. 23, 93-103.

Terpstra, C. and Wensvoort, G. (1987): Influence of the vaccination regime on the herd immune response for swine fever. Vet. Microbiol. 13, 143-151.

Terpstra, C. and Wensvoort, G. (1988): The protective value of vaccine-induced neutralising antibody titres in swine fever. Vet. Microbiol. 16, 123-128.

Terpstra, C., Bloemraad, M. and Gielkens, A. L. J. (1984): The neutralizing peroxidase-linked assay for detection of antibody against swine fever virus. Vet. Microbiol. 9, 113-120. 